\title{
La structure du récepteur cérébelleux de l'inositol triphosphate
}

L'inositol triphosphate (IP3), issu de l'hydrolyse de phospho-inositides membranaires par des phospholipases $C$, est un second messager universel découvert il y a un peu plus de six ans par Michael J. Berridge $e t$ al. Il stimule la libération de calcium d'organites intracellulaires vers le cytoplasme. Le réticulum endoplasmique semble constituer le site majeur de stockage du calcium, et donc l'organite cible de l'IP3. En 1988, S. Supattapone et al., du laboratoire de S.H. Snyder (Johns Hopkins University, Baltimore, MD, USA) rapportaient la purification à partir du cervelet d'une glycoprotéine de $260 \mathrm{kDa}$ ayant toutes les caractéristiques d'un récepteur de l'IP3, substrat possible de protéines kinases activées par l'AMP cyclique et pouvant se fixer à une lectine (la concanavaline A) [1].

Toutes ces propriétés semblaient étrangement rapprocher ce récepteur présomptif d'une autre protéine cérébelleuse, curieusement dénommée $\mathrm{P}_{400}$, étudiée par une équipe japonaise, dans un tout autre contexte, celui de souris mutantes ayant des signes d'ataxie. $\mathrm{P}_{400}$ apparaissait être en quantité très diminuée chez les souris atteintes [2] et être, chez l'animal normal, particulièrement abondante dans les cellules de Purkinje du cervelet. Ces cellules clés du traitement des informations transitant dans le cervelet sont également très riches en récepteurs pour l'IP3, comme l'indiquent les expériences d'autoradiographies des coupes de cervelet incubées avec de l'IP3 tritié. De fait, des vérifications complémentaires devaient démontrer l'identité de $\mathrm{P}_{400}$ et du candidat récepteur de $260 \mathrm{kDa}$. C.D. Ferris et al., du labo- ratoire de S.H. Snyder, viennent maintenant de démontrer que ce récepteur est également la molécule responsable des mouvements de calcium provoqués par l'IP3, alors qu'il eût été concevable que le récepteur fût physiquement distinct du canal calcique, couplé à lui, par exemple, par une G-protéine.

En effet, la molécule de récepteur purifiée, intégrée dans la membrane de vésicules lipidiques artificielles, possède les deux actions de liaison du médiateur et d'induction des mouvements transmembranaires de calcium [3]. Seuls l'ins $(1,4,5) \mathrm{P} 3$ et, quoique moins efficacement, l'ins $(2,4,5) \mathrm{P} 3$ sont d'actifs inducteurs des flux de calcium. L'ADN complémentaire codant pour le récepteur a été cloné en utilisant des anticorps dirigés contre la protéine $\mathrm{P}_{400}$. T. Furuichi et al., du laboratoire de K. Mikoshiba à Osaka (Japon), ont montré que ce récepteur possédait 2749 acides aminés, avec une région hydrophobe comportant plusieurs zones transmembranaires présomptives du côté carboxyterminal et une très large région hydrophile, probablement intracytoplasmique, du côté aminoterminal [2]. La principale particularité de ce récepteur est sa ressemblance avec le récepteur de la ryanodine des muscles striés, surtout dans sa région transmembranaire (figure 1). Le récepteur de la ryanodine est le canal calcique du réticulum sarcoplasmique, en interaction avec le récepteur voltage-dépendant sensible à la dihydropyridine et, par conséquent, élément clé du couplage entre excitation et contraction des fibres musculaires. Le récepteur d'IP3 semble exister normalement sous la forme d'un tétramère, d'un poids moléculaire voisin de $10^{6}[1,2,4]$, les quatre sous-unités pourraient ainsi être arrangées dans la membrane du réticulum endoplasmique de manière à ménager une structure de canal calcique à laquelle contribueraient les portions transmembranaires de chaque sous-unité ; la portion intracytoplasmique constituerait le site de fixation d'IP3, comportant aussi, probablement, des domaines jouant un rôle régulateur, par exemple en fonction de l'état de phosphorylation par des protéine kinases sensibles à l'AMPc (figure 1). L'ARN messager du récepteur est très abondant dans le cervelet normal, mais très diminué dans l'organe des souris mutantes ataxiques ayant une agénésie des cellules de Purkinje [2]. Les autres types de cellules, nerveuses ou autres, possèdent également le messager, mais en très faible concentration. Le récepteur semble associé au réticulum endoplasmique, mais aussi, quoique ces points soient plus discutés [4], à la membrane plasmique et à la membrane nucléaire. S'il en est bien ainsi, ce serait un argument contre l'hypothèse proposée par certains qu'existeraient des organites très spécialisés, cibles exclusives de l'IP3, appelés calciosomes [5].

Un récent article de R.D. Burgoyne et al., réalisé par des équipes anglaises de Liverpool, Cambridge et Southampton et par une équipe espagnole de Badajoz (en Extremadura) aboutit à des résultats sur les cellules chromaffines qui vont également dans un sens opposé à l'hypothèse faisant des calciosomes les organites spécialisés dans la réponse à l'IP3. Ces calciosomes ont en effet été caractérisés par la présence à leur niveau de calséquestrine, une protéine 


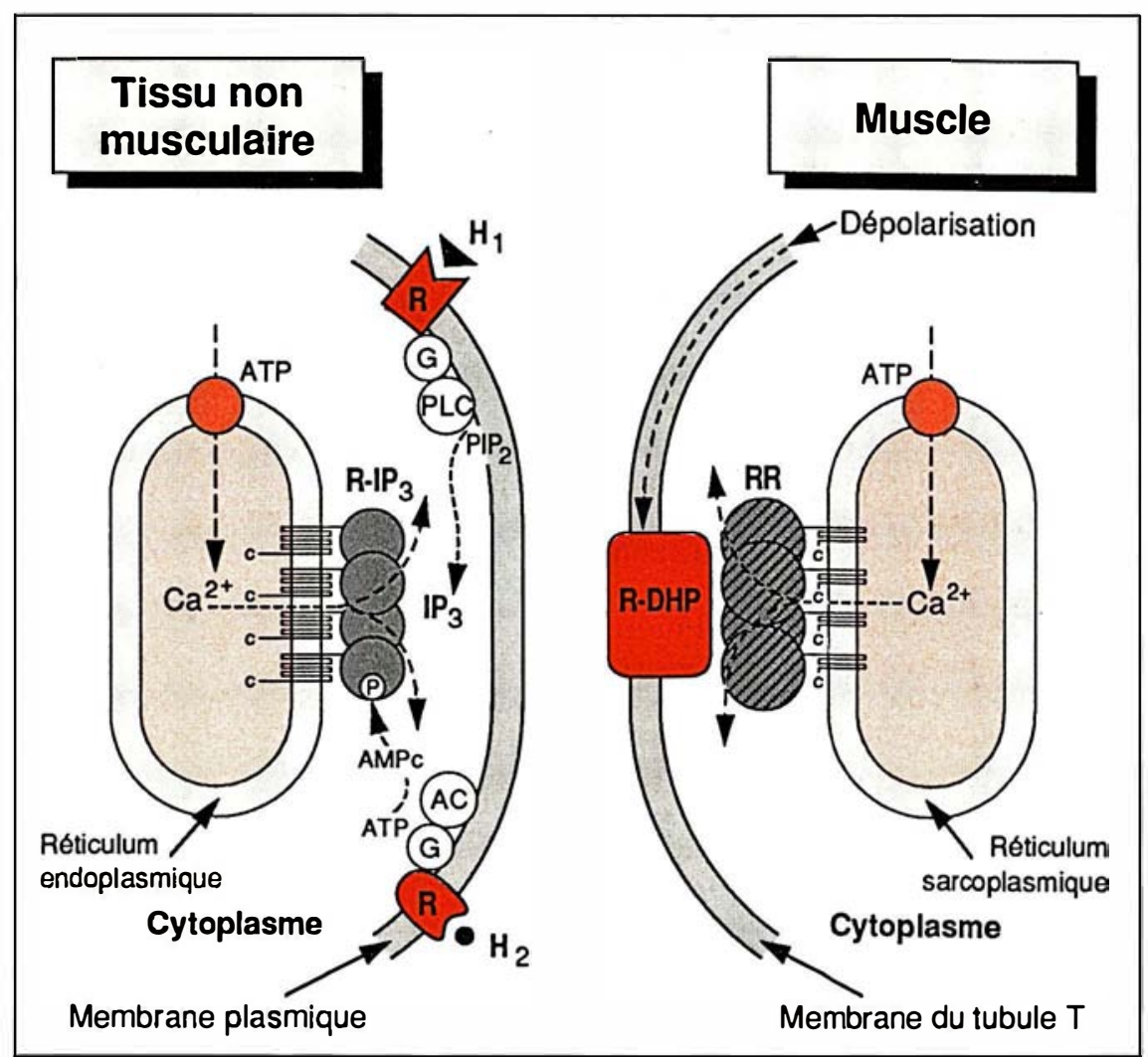

Figure 1. Comparaisons des voies de signalisation calcique utilisant le récepteur d'IP3 (R-IP3) et le récepteur de la ryanodine (RR) (d'après [4]). Dans des tissus non musculaires, des hormones $(H)$ se lient à des récepteurs $(R)$ qui peuvent être couplés à la phospholipase $C$ (PLC) ou à l'adénylate cyclase (AC) par des G-protéines (G). IP3 issu de I'hydrolyse du phosphatidylinositol 4,5 diphosphate (PIP2) se fixe à son récepteur, ce qui entraîne la sortie de calcium, préalablement stocké dans le réticulum endoplasmique grâce à l'action d'une pompe à calcium consommant de I'ATP. L'AMP cyclique synthétisé par l'adénylate cyclase active la phosphorylation du récepteur d'IP3, ce qui le désensibilise à l'action d'IP3. Dans le muscle, la dépolarisation membranaire induite par la transmission de l'influx nerveux est perçue par le récepteur de la dihydropyridine sensible au voltage. Le signal serait ensuite transmis au récepteur de la ryanodine dont l'extrémité $N$-terminale est en interaction avec la membrane du tubule $T$, au contact du récepteur de la dihydropyridine; cela entraîne l'activation du récepteurcanal et la sortie de calcium du réticulum sarcoplasmique. A noter la position des extrémités carboxyterminales des récepteurs-canaux, intraluminale dans le cas du récepteur d'IP3 et cytoplasmique dans le cas du récepteur de la ryanodine.

liant le calcium [3], et d'une ATPase dépendante du calcium, d'un poids moléculaire de 100000 . Or il semble que la cible de l'IP3 dans les cellules étudiées soit un organite différent de ceux possédant cette ATPase de $100 \mathrm{kDa}$, organite qui posséderait à la place une ATPase dépendante du calcium de $140 \mathrm{kDa}[6]$.

Les calciosomes apparaissent en revanche sensibles à la caféine, qui $\mathrm{m} / \mathrm{s} n^{\circ} 1$ vol. 6, janvier 90 récepteur, présent et actif dans les autres cellules, est identique à celui du cervelet ou bien - et cela semble peut-être plus probable - s'il existe, comme pour bien d'autres rẹ́cepteurs, des isoformes multiples.

A. $\mathbf{K}$.

1. Supattapone S, Worley PF, Baraban JM, Snyder SH. Solubilization, purification and characterization of an inositol, trisphosphate receptor. J Biol Chem 1988; 263 : 1530-4.

2. Furuichi T, Yoshikama S, Miyawaki A, Wada K, Macha N, Mikoshiba K. Primary structure and functional expression of the inositol 1,4,5 - triphosphate - binding protein $\mathrm{P}_{400}$. Nature $1989 ; 342: 32-8$.

3. Ferris C, Huganir RL, Supattapone S, Snyder SH. Purified inositol 1,4,5-triphosphate receptor mediates calcium flux in reconstituted lipid vesicles. Nature $1989 ; 342$ : 87-9.

4. Gill DL. Calcium signalling receptor kinships revealed. Nature $1989 ; 342: 16-8$.

5. Volpe P, Krause KH. Hashimoto S, et al. «calsiosome», a cytoplasmic organelle: the inositol 1,4,5-triphosphate sensitive $\mathrm{Ca}^{+}$ store of nonmuscle cells. Proc Natl Acad Sci USA 1988; 85 : 1091-5.

6. Burgoyne RD, Cheek TR, Morgan A, et al. Distribution of two distinct $\mathrm{Ca}^{2+}$ ATPaselikeproteins and their relationships to the agonist-sensitive calcium store in adrenal chromaffin cells. Nature $1989 ; 342$ : 72-4.

\section{BRÈVES}

口 $b c l-2$, un oncogène qui code pour une nouvelle G-protéine. Le gène $b c l-2$, localisé sur le chromosome 18, est activé lors des translocations $14 ; 18$ typiques de $85 \%$ des lymphomes folliculaires chez l'homme; il se trouve alors réarrangé avec la région des segments $\mathrm{J}$ des gènes de chaîne lourde d'immunoglobuline sur le chromosome 14. Le produit du gène, de $26 \mathrm{kDa}$, est localisé à la face interne de la membrane plasmique, est doué d'une activité de liaison du GTP et a une séquence protéique semblant le rattacher aux G-protéines [1] en ce qu'elle possède un site présomptif de liaison du GTP/GDP. On peut donc faire l'hypothèse que, probablement comme p2l ras, le produit du gène bcl-2 est impliqué, normalement, dans la transmission d'un signal mitogénique.

[1. Haldar S, et al. Nature $1989 ; 342$. 195-8.] 